\title{
Relationships following Neurological Conditions
}

The endeavour of understanding and supporting neurological conditions seems to have struggled within an historical paradox. The dominant paradigm of reducing down to the individual level (brain, self, individual emotional functioning, client-centred, etc.) has gained much (notably shining an increasingly bright spotlight on the qualities and complexities of subjective experience for those with neurological conditions). However this has been alongside the concurrent needs (and influences) of significant others connected to a person with a neurological condition within their tapestry of social relationships, from family outwards to different wider communities. These needs have historically not been responded to as 'core business' in many neurological services around the world, despite the voices of relatives, and other non-specialist agencies have become fractionated arenas where the needs of significant others may or may not be met.

The picture that emerges from these service trends is fractionated and punctuated as a result - too myopic to represent both the full health and social care costs of developmental, acquired and progressive neurological conditions across the lifespan for all connected in a social network, and the complex interconnected and reciprocal influences between people with neurological conditions and significant others. Perhaps a key construct that is most obscured by this frame is the space/processes between all people concerned: the relationships themselves. In my own clinical experience I have found a helpful and wellreceived conversation with relatives and friends that metaphorically describes the ripple of a problematic wave, emanating from the lesion in one person's brain, but passing through and disabling the minds of all concerned (this would of course include clinicians and the interpersonal challenges that we face in our work with our clients, particularly in longer-term work. We are not immune from the wave!).

But times are changing and we are in an exciting period of clinical-academic scholarship in the clinical neurosciences and related therapeutic traditions. The social neuroscience revolution no longer permits the view of isolated brains as valid, they are inherently social and this parameter cannot be abandoned in studying their function and dysfunction (importantly the latter prompts us to consider the direct impact of a lesion on another's mind via the intersubjective tapestry of connection). These developments have been unusually slow in translating over to intervention but this is progressing. The clinical rehabilitation and enablement traditions have necessarily broadened their theoretical tool-kits from neuroscience to theories that are inherently social, such as group psychotherapy, couples and family therapy, postmodern traditions such as social constructionism and sociolinguistics, critical social theory and community psychology. These applications have prompted and co-evolved with emerging clinical practices involving the client and their significant others. Excitingly, this emerging trend can be characterised as a move away from a rigid focus on independence to the rehabilitation/support of inter-dependence and the bonds/connections between people. Community and vocational rehabilitation services have necessarily had to involve large groups of people in contexts very distant from the neurology clinic.

I believe this special issue on relationships following neurological conditions across the lifespan is a fine example of these contemporary developments. As a counter-process to relationship-disconnection and breakdown following neuropathology, the following pages are a product of truly international and interdisciplinary collaboration and connection: the 
topic spans professional boundaries and geographical borders and as such should be a core concern for many. A range of approaches and methodologies are brought to bear on the complexity and diversity of relationship changes in neurological conditions. It is significant that relationships of all kinds are explored across the contributions: family (Helps; Gan and colleagues; Togher and colleagues; Tams and colleagues), couples (Kreutzer and colleagues), intimate relationships with self and others (Simpson and colleagues), friendships (Palmer \& Herbert), and work colleagues (Yeates and colleagues). The conditions represented include developmental (Helps), acquired in childhood (Gan and colleagues) and adulthood (Togher and colleagues; Simpson and colleagues; Palmer \& Herbert and Yeates and colleagues), and progressive (Tams and colleagues). A collection of papers such as this supports the idea that relationship challenges may be one of the most ubiquitous sequelae across neurological conditions, over a lifespan, and speaks to the interrelationship of neuropathology and human experience.
The data and ideas explored here offer direct suggestions for clinical work orientated to relationships of all kinds, and I hope readers will find value in the forthcoming pages. It has been a pleasure inviting and collating these submissions and I would like to thank the editors of NeuroRehabilitation for inviting me to act as a guest for this role. I am sure this will be a historically important collection of work, as part of the ongoing turn to the relational in neurorehabilitation and related fields.

Guest Editor

Giles Yeates

Principal Clinical Neuropsychologist Community Head Injury Service Buckinghamshire NHS PCT Jansel Square, Aylesbury HP21 7ET

UK

E-mail: Giles.Yeates@buckshealthcare.nhs.uk Tel.: +4401296 337760 Fax: +4401296337743. 